\title{
Thermodynamic properties of ultracold Bose gas: transition exponents and universality
}

\author{
Sanchari Goswami, Tapan Kumar Das \\ Department of Physics, University of Calcutta, 92 A.P.C. Road, Calcutta-700009, India \\ and \\ Anindya Biswas \\ Harish-Chandra Research Institute, Chhatnag Road, Jhunsi, Allahabad 211 019, India.
}

\begin{abstract}
We report exact numerical calculation of chemical potential, condensate fraction and specific heat of $N$ non-interacting bosons confined in an isotropic harmonic oscillator trap in one, two and three dimensions, as also for interacting bosons in a 3D trap. Quasi phase transitions are observed in all these cases, including one-dimension, as shown by a rapid change of all the thermodynamic quantities at the transition point. The change becomes more rapid as $N$ increases in 2D and 3D cases. However with increase in $N$, the sudden change in the nature of specific heat, gets gradually wiped out in $1 \mathrm{D}$, while it becomes more drastic in $2 \mathrm{D}$ and $3 \mathrm{D}$. The sudden change in the nature of condensate fraction and chemical potential as $N$ increases becomes more drastic even in 1D. Defining transition exponents, which characterize the nature of a thermodynamic quantity at the transition point of a quasi phase transition, we evaluate them by careful numerical calculation very near the transition temperature. These exponents are found to be independent of the size of the system and whether the bosons are interacting or not, demonstrating their universality property.
\end{abstract}

PACS numbers : 03.75.Hh, 64.60.Fr, 05.30.Jp, 75.40.Cx

\section{Introduction}

Bose-Einstein condensation (BEC) is the transition process, in which a macroscopic fraction of bosons goes into the lowest energy state, below a certain critical temperature $\left(T_{c}\right)$ [1]. It was predicted 
by Einstein in 1925, based on Bose's explanation of black body radiation and experimentally observed in harmonically trapped bosonic alkali atoms in 1995 (for details see Ref. [2]). BEC is generally considered as a phase transition in the thermodynamic limit, i.e. when the number of bosons $(N)$ goes to infinity. At a phase transition, thermodynamic quantities usually display a critical behavior. Hence it is essential to know how such quantities change at and across $T_{c}$. Unfortunately, their analytic expressions - known over many decades - are obtained under several idealized simplifying conditions, which are far from the actual laboratory situation. In some cases, the idealized treatment even gives wrong answers, as we will see below. Attempts have been made to partly relax the simplifying idealizations and use mathematical approximations to obtain analytic or semi-analytic expressions for thermodynamic quantities [8, 9, 10]. But a fully satisfactory analytic treatment is not possible, making a numerical investigation essential. In this communication, we present a numerical study of the critical behavior just across the BEC transition.

In standard text books 1, 3, it is shown that BEC is possible in an ideal (i.e. non-interacting) and homogeneous (i.e. not confined) Bose gas below a finite critical temperature $T_{c}^{0}$ (we use the superscript 0 to indicate the ideal case) in three and higher dimensions, while in two-dimension the critical temperature becomes zero and no BEC transition is possible in one-dimension. For a three-dimensional ideal and uniform Bose gas in the thermodynamic limit $(N \rightarrow \infty)$, the condensate fraction (defined as $\frac{N_{0}}{N}$, where $N_{0}$ is the number of bosons in the lowest energy state) and the heat capacity, at a temperature $T<T_{c}^{0}$, are given by [1, 4 ]

$$
\begin{array}{r}
\frac{N_{0}}{N}=1-\left(\frac{T}{T_{c}^{0}}\right)^{\frac{3}{2}}, \\
C_{N}(T)=\frac{15}{4} N k_{B} \frac{\zeta\left(\frac{5}{2}\right)}{\zeta\left(\frac{3}{2}\right)}\left(\frac{T}{T_{c}^{0}}\right)^{\frac{3}{2}},
\end{array}
$$

where $k_{B}$ is the Boltzmann constant and $\zeta(x)=\sum_{n=1}^{\infty} n^{-x}$ is the Riemann zeta function. It is assumed that the chemical potential $(\mu)$ remains zero for $T \leq T_{c}^{0}$, and starts to decrease above $T_{c}^{0}$. Above $T_{c}^{0}$, condensate fraction vanishes, while the heat capacity takes an involved expression (see Eq. (12.66) of Ref [1]). As functions of temperature, both condensate fraction and $C_{N}$ are continuous, while their first derivatives are discontinuous at $T=T_{c}^{0}$ (see Fig. 12.9 of Ref. [1]). This means that in this limit, BEC in an ideal and uniform Bose gas is a continuous phase transition process. The situation is quite different if the Bose gas is inhomogeneous, i.e., confined in a suitable trap [5]. Commonly used experimental traps are spherical or axially symmetric harmonic oscillator traps, for which $\frac{N_{0}}{N}=1-\left(\frac{T}{T_{c}^{0}}\right)^{3}$ and $C_{N}(T)$ has a finite discontinuity at $T=T_{c}^{0}$ [4, 1, 5] in the thermodynamic limit. Thus, the corresponding phase transition is of first order.

These analytic results are obtained under the following simplifying idealizations:

1. The derivation is done in the thermodynamic limit, while typical laboratory BECs contain a finite 
number of atoms.

2. The spacing between energy levels of the Bose gas is assumed to be much smaller than $k_{B} T$ for the temperatures under consideration, so that a sum over occupied energy levels can be replaced by an integral over energy. This semi-classical approximation is true only for an infinite, uniform system. However, if the condensate is confined in a finite trap (e.g. a harmonic trap), the level-spacing becomes appreciable and the approximation loses its validity. In the semi-classical approach, population of the lowest state is taken separately outside the integral, since the semi-classical density of states vanishes, while the Bose distribution function diverges at the lower limit.

3. The chemical potential $(\mu)$ is assumed to remain zero for $T \leq T_{c}^{0}$. However, when the sum is evaluated exactly for a finite $N, \mu$ (determined from the condition that the total number of bosons is $N$ ) decreases with increase of $T$, initially slowly from zero at $T=0$ up to $T=T_{c}$ and then rapidly for $T>T_{c}$.

4. Interatomic interactions are disregarded. In reality atoms interact through well known interatomic interactions. At the very low energy and temperature of a BEC, the $s$-wave scattering length $\left(a_{s}\right)$ governs the effective interaction. The latter can be repulsive as in ${ }^{87} \mathrm{Rb}$ and ${ }^{23} \mathrm{Na}$ atoms, or attractive as in ${ }^{7} \mathrm{Li}$ atoms. It is also possible to 'tune' the interaction to any desired value using the Feshbach resonance [6]. The interactions have a profound effect on the condensate properties, as also on $T_{c}$ [2]. In a harmonically trapped repulsive BEC, the hydrodynamic model shows that energy of most levels are lower than those of the corresponding non-interacting bosons [7]. Thus smaller thermal energy is needed to lift particles from the ground state. Consequently the critical temperature reduces appreciably.

Attempts have been made to remove some of these simplifying idealizations. Replacing the sum by an integration including semi-classical density of states, Bagnato and Kleppner [8] showed that BEC is possible in one-dimension, if the trap is more confining than a parabolic potential, while in two-dimension, $\mathrm{BEC}$ is possible for any power law confining potential. Using a modified semi-classical approximation, Yukalov 9] replaced the lower limit of integration from zero to a finite value given by the uncertainty relation. He thus removed the divergence at the lower limit of the integration to obtain Bose condensation for an arbitrary power law confinement in any dimension. Ketterle and van Druten [10] obtained analytic expressions for the condensate fraction and $T_{c}$, after relaxing the first two conditions: treating the sums appropriately for a finite $N$ and using some mathematical approximations. They demonstrated that BEC is possible in three, two and even one dimensional harmonic traps. This is in sharp contrast with the semi-classical treatment. The critical temperature in one dimension is higher than in three dimensional traps and therefore more convenient for experimental achievement of BEC in quasi-one dimensional traps. However, they still assumed $\mu=0$ for $T \leq T_{c}$ and considered only non-interacting 
bosons. All these attempts still retain some of the simplifying assumptions. Moreover, it is not always possible to get analytic expressions valid at $T_{c}$, approaching it both from below as also from above.

In the exact treatment of finite systems, in which sums are evaluated exactly, all thermodynamic quantities become continuous functions of $T$ [11, 12]. Consequently, there is no strictly critical temperature in such a system, although for large enough $N$, there is a distinct change in the nature of the curves over a small interval around a particular temperature, referred to as the transition temperature $\left(T_{c}\right)$. For very large $N$, the first derivatives tend to exhibit a discontinuity at $T_{c}$. Thus in the strict sense, there is no phase transition in a finite inhomogeneous system. The effects of semi-classical approximations in infinite systems and disregard of inter-particle interactions make $T_{c}^{0}$ appreciably larger than $T_{c}$.

For a true phase transition, one can define critical exponents, which characterize the nature of phase transition and its universal properties [13. When the transition from the condensed phase to the Bose gas phase is gradual and there is no true phase transition, the critical exponents vanish. Still, for a large enough $N$. there is a sharp enough transition, which we refer to as quasi phase transition (QPT). In this case, we define a transition exponent (TE), which shows the characteristic nature of the thermodynamic quantity across the transition temperature. In this work, we evaluate and examine the transition exponents (defined in the next Section) to provide an understanding of the nature of thermodynamical quantities near a quasi phase transition. Since a laboratory condensate contains interacting atoms, we also investigate interacting condensates. Inclusion of interatomic interactions, makes the many-body problem non-trivial. We use the correlated potential harmonic expansion method (CPHEM) [14 for solving the interacting many-body system.

The paper is organized as follows. Sec. II presents the theoretical background, providing definitions and basic equations. Sub-section II.A defines the transition exponent for a quasi phase transition. In the next sub-section we discuss how thermodynamic quantities and TE can be evaluated for a finite system. A third sub-section is included to briefly outline the CPHEM. Sec. III presents the results of our calculation and finally we draw our conclusions after a brief summary in Sec. IV.

\section{Theoretical background}

\section{II.1 Transition exponent for a quasi phase transition}

An important physical quantity in connection with a true phase transition at a critical point is the critical exponent. A critical point is characterized by divergences or discontinuities in thermodynamic quantities, depending on the nature of the thermodynamic quantity and dimensionality of the system. 
Thus the thermodynamic quantity may cease to be analytic at the critical point. The critical exponent is useful in understanding the rapidly changing behavior of thermodynamic functions at the critical temperature $\left(T_{c}^{0}\right)$. In terms of a reduced temperature

$$
t=\frac{T-T_{c}^{0}}{T_{c}^{0}}
$$

which is a dimensionless measure of the deviation of temperature from the critical temperature, one can define the critical exponent $(\lambda)$ for a thermodynamic function $F(t)$ as 13 .

$$
\lambda=\lim _{t \rightarrow 0} \frac{\ln |F(t)|}{\ln |t|} .
$$

This corresponds to $F(t) \sim|t|^{\lambda}$, for small values of $|t|$. Obviously, at the critical temperature, $F(t)$ must either vanish or be singular, depending on the sign of $\lambda$ (for $\lambda \neq 0$ ). But sometimes (particularly in a QPT) thermodynamic quantities are neither singular, nor do they vanish at the transition temperature. A typical example is the heat capacity of a BEC, either a finite one or in the thermodynamic limit. In such cases, one can take $F(t)$ to be the difference of the desired thermodynamic function at $T$ and its value at $T_{c}$. Indeed, in this case, $\lambda$ is zero and the following prescription will give $\lambda_{1}$ (which must be positive) of the relation 13 .

$$
F(t)=F(0)+b|t|^{\lambda_{1}}+\ldots .
$$

Since in this case, $\lambda$ is zero, $\lambda_{1}$ is the leading exponent of interest. The nature of the thermodynamic function near the critical point is determined by $\lambda_{1}$. Hence for such a situation, we define $\lambda_{1}$ as the transition exponent, given by

$$
\lambda_{1}=\lim _{t \rightarrow 0} \frac{\ln |F(t)-F(0)|}{\ln |t|} .
$$

For $T<T_{c}^{0}$, the condensate fraction for non-interacting bosons in the thermodynamic limit is $\frac{N_{0}}{N}=$ $1-\left(\frac{T}{T_{c}^{0}}\right)^{\alpha}$, where $\alpha=\frac{3}{2}$ for a uniform system, while $\alpha=3$ for bosons in a 3-D harmonic trap. Then it is easy to see that $\lambda=1$. For the heat capacity, $C_{N}(T) /\left(\alpha N k_{B}\right)=a\left(T / T_{c}^{0}\right)^{\alpha}$ (a being a constant) and one has $\lambda=0$. On the other hand, chemical potential remains zero for $T \leq T_{c}^{0}$ and for $T>T_{c}^{0}$ it is obtained numerically even in the thermodynamic limit [1]. Hence no closed analytic form is possible and numerical analysis is the only possibility. Thus for chemical potential $\lambda$ is undefined for $T<T_{c}^{0}$ and has to be obtained numerically for $T>T_{c}^{0}$, even in the thermodynamic limit.

For QPT with $N$ small, the thermodynamic functions are smooth across the transition region. If the function does not have an extremum at $T_{c}$ [like chemical potential and condensate fraction in 1-D, 2-D and 3-D and specific heat in 1-D (see later)] a simple Taylor series expansion shows that $\lambda=0$ and $\lambda_{1}=1$. On the other hand heat capacity in 2-D and 3-D (for both interacting and non-interacting bosons) has a maximum and one sees that $\lambda=0$ while $\lambda_{1}=2$. But in the $N \rightarrow \infty$ limit, exact numerical calculation shows a sharp fall in the value of $C_{N}(T)$. For a mathematical discontinuity, $\lambda_{1}$ may 
be different from 2, and may have different values for $T<T_{c}$ and for $T>T_{c}$. Thus it is interesting to calculate $\lambda_{1}$ numerically for a large enough value of $N$, to understand how the thermodynamic function changes across the transition region, as $N$ increases.

An important characteristic property is the universality of the critical exponent. The value of $\lambda$ does not depend on the interatomic interaction or detailed nature of the system. Its value depends only on the dimension of the system and the symmetry of the order parameter [13. We expect a similar universality property satisfied by the transition exponents $\left(\lambda_{1}\right)$ as well. In the present work, we calculate transition exponents for a number of thermodynamic functions of the BEC in different cases and investigate whether they depend on the system parameters. We consider a non-interacting Bose gas trapped in a harmonic oscillator potential in one, two and three dimensions. Finally, we also include realistic interatomic interactions in an approximate many-body treatment of the real Bose gas. As discussed earlier, thermodynamic functions like specific heat, condensate fraction and chemical potential depend on the dimension of the system, the choice of the trap potential, whether interatomic interactions are included or not, etc. But transition exponents extracted from them may display a universal property, similar to that exhibited by the critical exponents in a true phase transition. Our present work aims to explore whether the transition exponents exhibit an underlying universality.

\section{II.2 System of non-interacting bosons}

In this sub-section, we consider $N$ non-interacting bosonic atoms trapped in an isotropic harmonic potential of frequency $\omega$ in $d$-dimensional space $(d=1,2,3)$. The energy scale is so chosen that the single particle ground state is at zero energy. The energy eigenvalues $E_{n}(n=0,1,2, \ldots)$ are given by

$$
E_{n}=n \hbar \omega
$$

The number of particles in the $n$-th state with energy $E_{n}$ at a temperature $T$ is given by the Bose distribution function

$$
f\left(E_{n}\right)=\frac{1}{e^{\beta\left(E_{n}-\mu\right)}-1}
$$

where $\beta=1 / k_{B} T$ and $\mu$ is the chemical potential. The latter is determined from the constraint that the total number of particles is $N$

$$
N=\sum_{n=0}^{+\infty} \gamma_{n} f\left(E_{n}\right)
$$

where $\gamma_{n}$ is the degeneracy of the $n$-th level. It is $1,(n+1)$ and $\frac{(n+1)(n+2)}{2}$ for the one-, two- and three-dimensional harmonic oscillator respectively. Clearly, $\mu$ has a temperature dependence. The total 
energy for the system is given by

$$
E(N, T)=\sum_{n=0}^{+\infty} \gamma_{n} f\left(E_{n}\right) E_{n}
$$

The specific heat for fixed particle number $(N)$ is calculated using the relation

$$
C_{N}(T)=\frac{\partial E(N, T)}{\partial T}
$$

Using (7), (91), (10), one can obtain the heat capacity as

$$
\begin{gathered}
C_{N}(T)=\beta \sum_{n=0}^{+\infty} \frac{\gamma_{n} E_{n} \exp \left(\beta\left(E_{n}-\mu\right)\right)}{\left.\exp \left(\beta\left(E_{n}-\mu\right)\right)-1\right)^{2}}\left[\frac{E_{n}-\mu}{T}+\frac{\partial \mu}{\partial T}\right] \\
=\beta \sum_{n=0}^{+\infty} \frac{\gamma_{n} E_{n} \exp \left(\beta\left(E_{n}-\mu\right)\right)}{\left(\exp \left(\beta\left(E_{n}-\mu\right)\right)-1\right)^{2}}\left[\frac{E_{n}-\mu}{T}-\frac{\sum_{m=0}^{+\infty} \gamma_{m}\left(E_{m}-\mu\right) \exp \left(\beta\left(E_{m}-\mu\right)\right)\left(f\left(E_{m}\right)\right)^{2}}{T \sum_{p=0}^{+\infty} \gamma_{p} \exp \left(\beta\left(E_{p}-\mu\right)\right)\left(f\left(E_{p}\right)\right)^{2}}\right] .
\end{gathered}
$$

In the semi-classical treatment, $\mu$ is assumed to remain zero for $T \leq T_{c}^{0}$ and start decreasing for $T>T_{c}^{0}$. But a numerical solution of Eq. (8) for a finite $N$ shows that $\mu$ decreases very slowly from its maximum value (zero) at $T=0$, as $T$ increases in the interval $T \leq T_{c}$. The rate of decrease becoming suddenly rapid at the transition temperature $T_{c}$. Thus, in this case there is no sharp critical temperature. The heat capacity also becomes a smooth function of $T$, attaining a maximum at a temperature, at which $\mu$ suddenly becomes a rapidly decreasing function of $T$ (except for the one-dimensional case, see below). The transition temperature $T_{c}$ is usually defined as the temperature at which $C_{N}(T)$ is a maximum [1]

$$
\left.\frac{\partial C_{N}(T)}{\partial T}\right|_{T_{c}}=0 .
$$

We use our definition of TE, Eq. (5), separately for $T>T_{c}$ and $T<T_{c}$ (denoted by the superscripts + and - respectively), for different thermodynamic functions $(X)$

$$
\lambda_{1}^{X, \pm}=\lim _{t \rightarrow 0 \pm} \frac{\ln \left|F^{X}(t)-F^{X}(0)\right|}{\ln |t|},
$$

where $t=\left(T-T_{c}\right) / T_{c}$ and $X$ stands for chemical potential (chempot), condensate fraction (condfrac) and specific heat (spht) and $F^{X}(t)$ is $\mu(t), \frac{N_{0}}{N}(t)$ and $C_{N}(t)$ respectively. For all thermodynamic functions, we use the same transition temperature defined by Eq. (12).

\section{II.3 Realistic interacting bosons}

For the interacting case, one has to solve the many-body Schrödinger equation. An essentially exact solution is possible by the diffusion Monte Carlo (DMC) method [15. However, this has been done only for $N$ less than $\sim 100$. The mean-field approach together with the assumption of a contact twobody interaction leads to the commonly used Gross-Pitaevskii equation (GPE) [2]. In this approach, all correlations are disregarded and no realistic two-body interaction can be used. In addition, for an attractive BEC, there is a pathological singularity at the origin [16. Hence, we adopt a simplified fewbody technique, called correlated potential harmonics expansion method (CPHEM) [17, 14], in which 
all two-body correlations are retained, but higher-body correlations are neglected. Disregard of higherorder correlations is manifestly justified for a laboratory BEC, since it is designed to be so dilute that three-body collisions do not take place, to preclude formation of molecules and consequent depletion of the condensate through thee-body recombination. Furthermore, any realistic interatomic interaction can be incorporated in the CPHEM. This technique has been successfully applied to both repulsive and attractive BECs [18].

In the following, we review the CPHEM very briefly. Interested readers can get the details from Refs. [17, 14]. Schrödinder equation for the relative motion of a system of $N$ identical spinless bosons, interacting via pair-wise potential $V$ and trapped by $V_{\text {trap }}$ is

$$
\left[-\frac{\hbar^{2}}{m} \sum_{i=1}^{\mathcal{N}} \nabla_{\vec{\zeta}_{i}}^{2}+V_{\text {trap }}\left(\vec{\zeta}_{1}, \ldots, \vec{\zeta}_{\mathcal{N}}\right)+V\left(\vec{\zeta}_{1}, \ldots, \vec{\zeta}_{\mathcal{N}}\right)-E_{R}\right] \psi\left(\vec{\zeta}_{1}, \ldots, \vec{\zeta}_{\mathcal{N}}\right)=0
$$

where $\left\{\vec{\zeta}_{1}, \vec{\zeta}_{2}, \ldots, \vec{\zeta}_{\mathcal{N}}\right\}$ is the set of $\mathcal{N}=(N-1)$ Jacobi vectors, which are the relative variables, after separation of the center of mass motion [19] and $E_{R}$ is the energy of the relative motion. A global length called 'hyperradius' is defined as

$$
r=\left[\sum_{i=1}^{\mathcal{N}} \zeta_{i}^{2}\right]^{\frac{1}{2}}
$$

This, together with a set of $(3 \mathcal{N}-1)$ 'hyperangles', constituted by $2 \mathcal{N}$ polar angles of $\mathcal{N}$ Jacobi vectors and $(\mathcal{N}-1)$ angles defining their relative lengths [19] define the hyperspherical variables, replacing the Jacobi vectors. These are $3 \mathcal{N}$-dimensional analogue of 3 -dimensional spherical polar coordinates. Likewise, the $3 \mathcal{N}$-dimensional analogue of spherical harmonics are the hyperspherical harmonics $(\mathrm{HH})$. These are the eigenfunctions of the grand orbital operator, which is the hyperangular part of the $3 \mathcal{N}$ dimensional Laplace operator $\sum_{i=1}^{\mathcal{N}} \nabla_{\vec{\zeta}_{i}}^{2}[19$. It is natural to expand $\psi$ in the complete set of $\mathrm{HH}$, giving rise to the hyperspherical harmonics expansion method (HHEM). But the degeneracy of the HH basis increases very rapidly with $N$. Consequently, imposition of symmetry and calculation of matrix elements become practically impossible for $N>3$. Use of the full $\mathrm{HH}$ basis for the expansion of $\psi$ includes all many-body correlations in the wave function. However as mentioned earlier, a typical laboratory BEC is designed to be physically very dilute, since otherwise three-body collisions will lead to formation of molecules and consequent depletion of the condensate [2]. This means that three- and higher-body correlations are negligible in such condensates. Hence $\psi$ can be decomposed into interacting-pair Faddeev components, $\psi_{i j}$ (which becomes a function of the $\left(i j\right.$ )-pair separation $\vec{r}_{i j}$ and hyperradius $r$ only, due to neglect of higher than two-body correlations)

$$
\psi=\sum_{i, j>i}^{N} \psi_{i j}\left(\vec{r}_{i j}, r\right)
$$

Then, instead of the full HH basis, one can choose a subset, called potential harmonics (PH) subset 20] for the expansion of $\psi_{i j}$. The PH subset is defined as the subset of HH necessary for the expansion of 
the two-body interaction, $V\left(\vec{r}_{i j}\right)$. Since $\psi_{i j}$ is a function of $\vec{r}_{i j}$ and $r$ only, the PH basis is sufficient for its expansion, which reads

$$
\psi_{i j}\left(\vec{r}_{i j}, r\right)=r^{-\frac{(3 \mathcal{N}-1)}{2}} \sum_{K} \mathcal{P}_{2 K+l}^{l m}\left(\Omega_{\mathcal{N}}^{i j}\right) u_{K}^{l}(r)
$$

where $\mathcal{P}_{2 K+l}^{l m}\left(\Omega_{\mathcal{N}}^{i j}\right)$ is a potential harmonic [20]. The $r$-dependent factor in front is included to remove the first derivative with respect to $r$. Although each $\mathrm{HH}$ is in general a function of all $3 \mathcal{N}-1$ hyperangles, $\Omega_{\mathcal{N}}^{i j}$, the PH, being a subset of HH sufficient for the expansion of $V\left(\vec{r}_{i j}\right)$, is a function of only three hyperangles: polar angles of $\vec{r}_{i j}$ and a hyperangle $(\phi)$ defined through $r_{i j}=r \cos \phi$. Corresponding quantum numbers are $l, m$ and $K$. This corresponds physically to freezing all irrelevant degrees of freedom, and setting corresponding quantum numbers to zero. The physical picture is that when the ( $i j$-pair interacts, rest of particles are inert spectators and do not contribute to orbital and grand-orbital angular momenta. Thus the orbital angular momentum of the system is contributed by the interacting pair only [17, 14]. Substitution of the expansion, Eq. (17) in the Faddeev equation for the (ij)-partition

$$
\left(-\frac{\hbar^{2}}{m} \sum_{i=1}^{\mathcal{N}} \nabla_{\vec{\zeta}_{i}}^{2}+V_{\text {trap }}-E_{R}\right) \psi_{i j}=-V\left(r_{i j}\right) \sum_{k, l>k}^{N} \psi_{k l},
$$

and projection on the $\mathrm{PH}$ corresponding to the $(i j)$-partition give a set of coupled differential equations in $r$. Any suitable interatomic potential can be chosen for $V\left(\vec{r}_{i j}\right)$. Realistic potentials have a strong repulsion at very short separations. Hence $\psi_{i j}$ should be extremely small at such separations. On the other hand, the leading potential harmonics (corresponding to $K=0$ and small $K$ values) of Eq. (17) are appreciably large for small $r_{i j}$, resulting in a very slow rate of convergence. This is corrected by inclusion of an additional correlation function, $\eta\left(\vec{r}_{i j}\right)$, which simulates the nature of $\psi_{i j}\left(\vec{r}_{i j}, r\right)$ for small $r_{i j}$

$$
\psi_{i j}\left(\vec{r}_{i j}, r\right)=r^{-\frac{(3 \mathcal{N}-1)}{2}} \sum_{K} \mathcal{P}_{2 K+l}^{l m}\left(\Omega_{\mathcal{N}}^{i j}\right) u_{K}^{l}(r) \eta\left(\vec{r}_{i j}\right) .
$$

Note that the energy of the interacting pair in the condensate is negligibly small compared with the energy scale of the interatomic interaction. Hence at small values of $r_{i j}, \psi_{i j}$ should behave as the zero energy solution, $\eta\left(\vec{r}_{i j}\right)$, of the interacting pair

$$
-\frac{\hbar^{2}}{m} \frac{1}{r_{i j}^{2}} \frac{d}{d r_{i j}}\left(r_{i j}^{2} \frac{d \eta\left(r_{i j}\right)}{d r_{i j}}\right)+V\left(r_{i j}\right) \eta\left(r_{i j}\right)=0
$$

With the addition of this intuitive short-range correlation function, rate of convergence is dramatically improved. Moreover, the asymptotic form of $\eta\left(\vec{r}_{i j}\right)$ is $C\left(1-a_{s} / r_{i j}\right)$, which depends on the $s$-wave scattering length $\left(a_{s}\right)$ [4. Hence the short-range repulsion of the realistic two-body potential can be adjusted to correspond to the appropriate $a_{s}$ (specifying the effective two-body interaction) for the chosen condensate. 
Substitution of the expansion, Eq. (19) in Eq. (18) followed by projection on the PH corresponding to the $(i j)$-partition give a set of coupled differential equations (CDE)

$$
\begin{aligned}
{\left[-\frac{\hbar^{2}}{m} \frac{d^{2}}{d r^{2}}+\frac{\hbar^{2}}{m r^{2}}\{\mathcal{L}(\mathcal{L}+1)\right.} & \left.+4 K(K+\alpha+\beta+1)\}+V_{\text {trap }}(r)-E_{R}\right] U_{K l}(r) \\
& +\sum_{K^{\prime}} f_{K l} V_{K K^{\prime}}(r) f_{K^{\prime} l} U_{K^{\prime} l}(r)=0
\end{aligned}
$$

where $U_{K l}(r)=f_{K l} u_{K}^{l}(r), \mathcal{L}=l+\frac{3 N-6}{2}, \alpha=\frac{3 N-8}{2}, \beta=l+\frac{1}{2} . l$ is the orbital angular momentum of the condensate. The constant $f_{K l}^{2}$ is the overlap of the $\mathrm{PH}$ for interacting partition with the sum of PHs corresponding to all partitions [20. The correlated potential matrix element $V_{K K^{\prime}}(r)$ is given by [14]

$$
V_{K K^{\prime}}(r)=\left(h_{K}^{\alpha \beta} h_{K^{\prime}}^{\alpha \beta}\right)^{-\frac{1}{2}} \int_{-1}^{+1} P_{K}^{\alpha \beta}(z) V\left(r \sqrt{\frac{1+z}{2}}\right) P_{K^{\prime}}^{\alpha \beta}(z) \eta\left(r \sqrt{\frac{1+z}{2}}\right) W_{l}(z) d z,
$$

where $h_{K}^{\alpha \beta}$ and $W_{l}(z)$ are respectively the norm and weight function [21] of the Jacobi polynomial $P_{K}^{\alpha \beta}(z)$. Since $\eta\left(\vec{r}_{i j}\right)$ is included, the expansion basis is no longer orthogonal. One can follow standard procedure for non-orthogonal basis. However, dependence on $r$ of the overlap matrix complicates this procedure. On the other hand, we found that $\eta\left(r_{i j}\right)$ obtained numerically from Eq. (20) differs appreciably from a constant value only in a very small interval of small $r_{i j}$ values. Hence $<\mathcal{P}_{2 K+l}^{l m}\left(\Omega_{N}^{(i j)}\right) \mid \mathcal{P}_{2 K+l}^{l m}\left(\Omega_{N}^{(k l)}\right) \eta\left(r_{k l}\right)>$ is nearly independent of $r$. Disregarding its derivatives we approximately get Eq. (21), with $V_{K K^{\prime}}(r)$ given by Eq. (22). The fact that the overlap is not one is taken through the asymptotic constant $C$ of $\eta\left(r_{i j}\right)$. This implies that pairs of atoms at very low energy interact via an effective interaction $V\left(r_{i j}\right) \eta\left(r_{i j}\right)$. This can be understood as follows. Atoms having a very large de Broglie wave length at extremely low energy cannot come too close to feel the actual atom-atom potential, which is very strong. In this limit the total scattering cross section is $4 \pi\left|a_{s}\right|^{2}$ and $a_{s}$ specifies the effective atom-atom interaction in the zero energy limit [4, 2]. A fairly fast computer code can solve Eq. (21) using the hyperspherical adiabatic approximation [22] with upto 15000 particles in the condensate. This technique has been tested against known results, both experimental ones and theoretical ones calculated by other authors, for repulsive as well as attractive condensates [17, 14, 18. The realistic van der Waals (vdW) potential is used to obtain a large number of energy eigenvalues of the entire BEC for different orbital angular momenta of the system. These are then used in Eq.(8) to calculate the chemical potential at a chosen temperature $T$. Note that in this case, the sums over $n$ in Eqs, (8), (9) and (11) are to be replaced by double sums over $\{n, l\}$ and $\gamma_{n}$ is replaced by $(2 l+1)$. The energy eigenvalue $E_{n l}$ is the energy of the $n$-th excitation for the orbital angular momentum $l$ of the system. Finally Eq. (11) is used to calculate $C_{N}(T)$ [12]. Condensate fraction is obtained as $\frac{N_{0}}{N}$, where $N_{0}$ is the $(n=0, l=0)$ term of Eq.(8). 


\section{Results}

\section{III.1 Non-interacting bosons}

In this sub-section, we consider $N$ non-interacting bosons in an isotropic harmonic trap in one, two and three dimensions. We first present the results for the one dimensional case. A plot of condensate fraction against $k_{B} T / \hbar \omega$ shows a fast and almost linear drop for a considerable stretch, followed by a slowly decreasing part gradually going to zero as is shown in Fig. 1. The change over from the sharp linear drop to the gradually decreasing portion is fairly sudden for large $N$. This shows that a QPT occurs, in agreement with Ketterle and van Druten [10, and in contradiction with semi-classical treatment in text books. In Ref. [10], chemical potential was taken to be zero for $T \leq T_{c}$. This is strictly true in the thermodynamic limit only. When $N$ is finite, $\mu$ decreases slowly for $T \leq T_{c}$, then rapidly for $T>T_{c}$. In this case, $C_{N}(T)$ is a monotonically increasing function (see below).

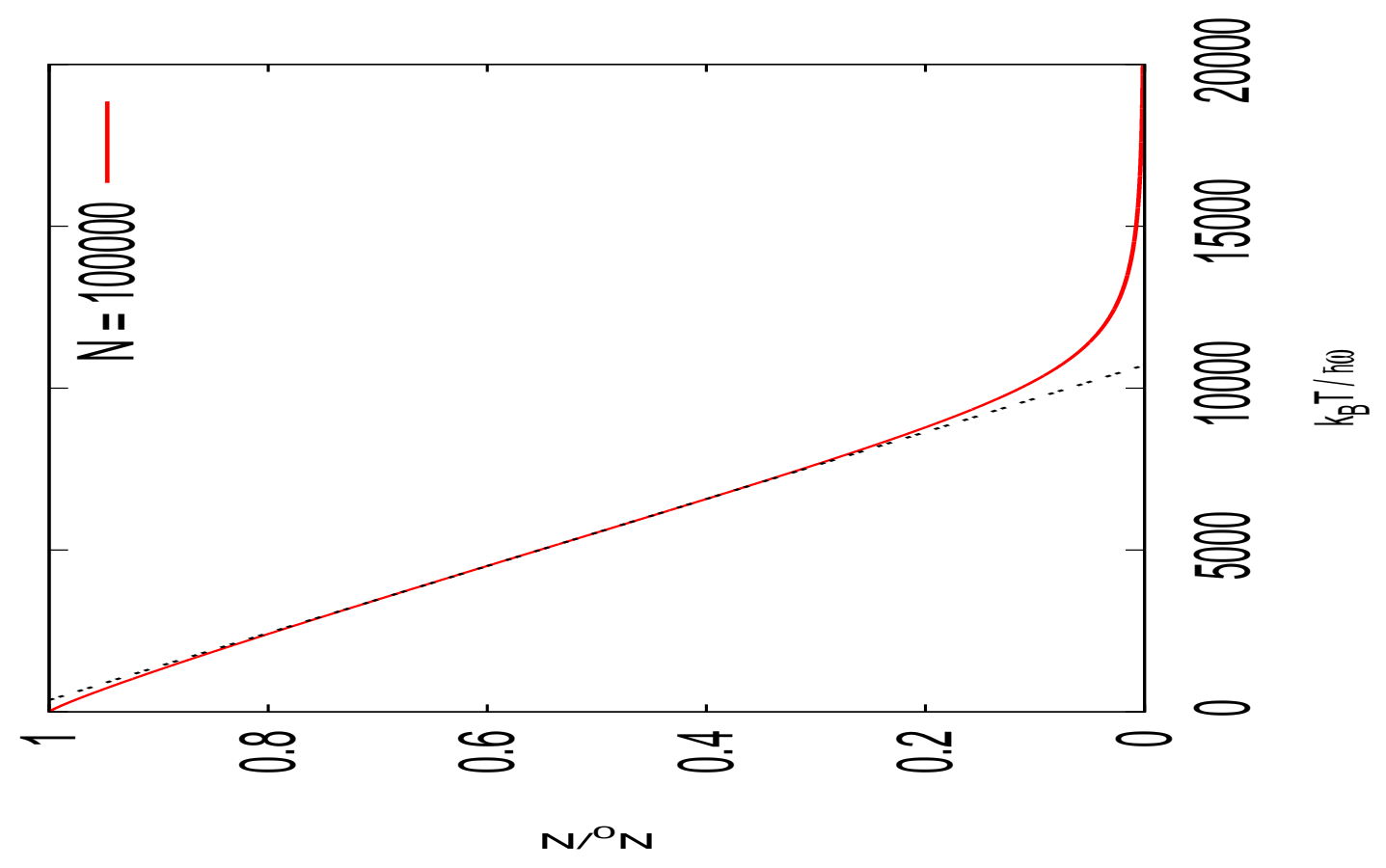

Figure 1: (Color online) Plot of condensate fraction as functions of $k_{B} T / \hbar \omega$ for 100000 non-interacting bosons in a one-dimensional harmonic trap. The extrapolated straight portion with the horizontal axis of condensate fraction versus $k_{B} T / \hbar \omega$ plot,as shown by the black dotted line gives the transition temperature $k_{B} T_{c} / \hbar \omega$.

In the absence of definition Eq. (12), we take the transition temperature $\left(k_{B} T_{c} / \hbar \omega\right)$ to be the intercept of the extrapolated straight portion with the horizontal axis of condensate fraction versus $k_{B} T / \hbar \omega$ plot. This value is somewhat larger than that in Ref. [10. In Table I, we present calculated values of $k_{B} T_{c} / \hbar \omega$ for different $N$, together with the the values obtained from a numerical solution of Eq. (16) of Ref. [10]. 
It is seen that the percentage difference is fairly large for small $N$ and decreases with increasing $N$.

Table 1: BEC transition temperature $\left(T_{c}\right)$ for non-interacting bosons in one dimensional trap.

\begin{tabular}{|c|c|c|}
\hline \multirow{2}{*}{$N$} & \multicolumn{2}{|c|}{$k_{B} T_{c} / \hbar \omega$} \\
\cline { 2 - 3 } & Calculated & From Ref. [10] \\
\hline 1000 & 196.0 & 171.3 \\
10000 & 1381.1 & 1274.9 \\
100000 & 10713.6 & 10088.4 \\
\hline
\end{tabular}

We next present the thermodynamic quantities as functions of $T / T_{c}$. In panel (a) of Fig. 2, we plot $\mu / \hbar \omega$ against $T / T_{c}$ for $N=1000,10000$ and 100000. One notices that as temperature increases, $\mu$ remains nearly constant for $T / T_{c}$ less than about 1 , after which it decreases rapidly. The change over in the nature of the decrease around $T_{c}$ becomes sharper as $N$ increases. This again clearly demonstrates the occurrence of a QPT at $T_{c}$. In panel (b) of Fig. 2, we plot the condensate fraction, as a function of $T / T_{c}$ for the chosen values of $N$. As stated earlier, we notice that a rapid change in the rate of decrease occurs at around $T_{c}$, the change being sharper as $N$ increases. This demonstrates that a BEC phase is possible and it goes gradually over to the normal Bose gas phase. In panel (b), we also include the straight line $-1 * x+1$, with $x=T / T_{c}$. The curves overlap more and more with this straight line over a considerable region as $N$ increases. However, from a plot of $C_{N} / N k_{B}$ against $T / T_{c}$ in panel (c), we notice that for the one-dimensional case, $C_{N}(T)$ is a monotonically increasing function of $T$ This implies that the one-dimensional case is distinctly different from higher dimensions. However, we notice from Fig. 2(c), that $C_{N}(T)$ is almost linear for $T$ far away from the transition region, with distinctly different slopes. The difference of slopes decreases as $N$ increases. Consequently, criticality in the nature of $C_{N}(T)$ gets smeared out as $N$ increases for one dimensional case.

Standard text book treatments, replacing sums by integrals and assuming $\mu=0$ for $T \leq T_{c}$, in the thermodynamic limit, conclude that BEC is not possible in one-dimension. Our calculations treating the sums exactly and allowing $\mu$ to take appropriate value at all temperatures, show that a QPT is possible even in one dimension for a finite number of bosons. This is in agreement with Ketterle and van Druten [10] who pointed out that BEC is possible in one dimension at a higher transition temperature. But our calculations also show that in the limit $N \rightarrow \infty$, the rapid change in $C_{N}(T)$ across the transition temperature gradually fades away.

Using $T_{c}$ obtained above, we plot $\ln \left|\left(\mu(T)-\mu\left(T_{c}\right)\right) / \hbar \omega\right|, \ln \left|\frac{N_{0}(T)}{N}-\frac{N_{0}\left(T_{c}\right)}{N}\right|$ and $\ln \mid\left(C_{N}(T)-\right.$ 


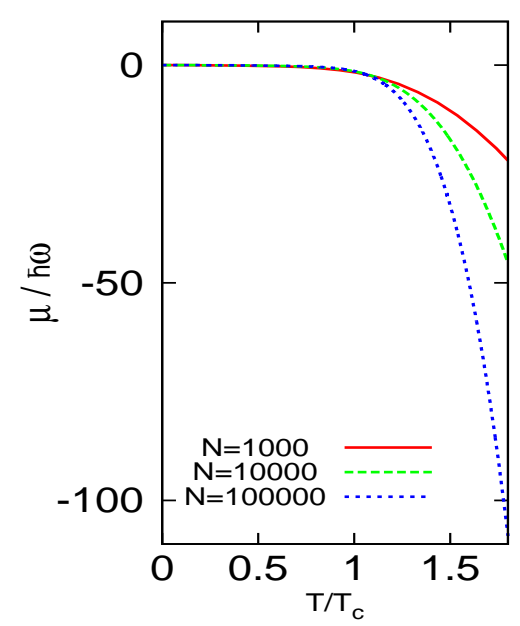

(a)

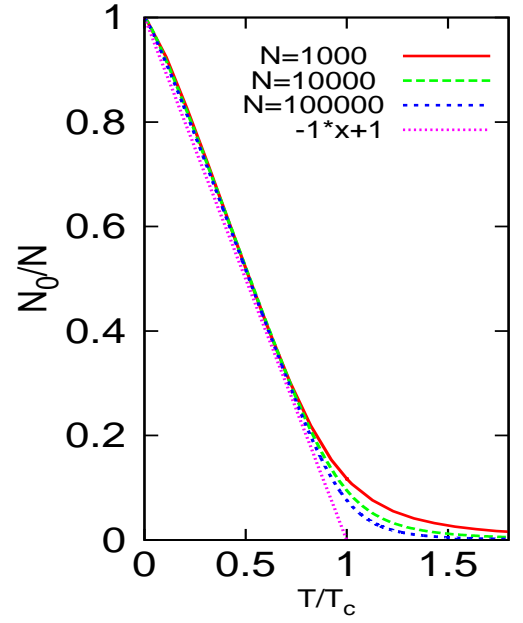

(b)

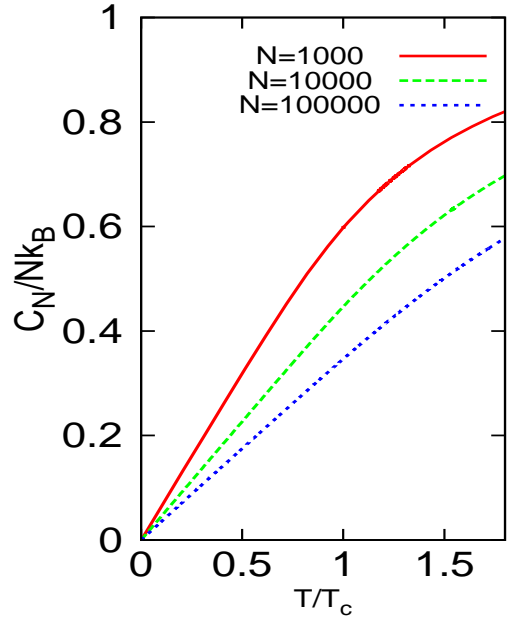

(c)

Figure 2: (Color online) Plot of chemical potential (panel a), condensate fraction (panel b) and $C_{N} / N k_{B}$ (panel c) as functions of $T / T_{c}$ for indicated number $(N)$ of non-interacting bosons in a one-dimensional harmonic trap. Note that chemical potential is expressed in energy oscillator unit $(\hbar \omega)$. Note also that $C_{N} / N k_{B}$ is a monotonically increasing function of $T$, giving rise to the common notion that there is no criticality in one dimension.

$\left.C_{N}\left(T_{c}\right)\right) / N k_{B} \mid$ against $\ln |t|$, for $t<0$, in panels (a), (b) and (c) of Fig. 3 respectively. As expected, we obtain straight line fits for small $|t|(\ln |t|<-4)$. All such straight line fits for different $N$ are parallel (to within numerical errors), showing that the transition exponents are independent of $N$. A straight line fit for the largest $N$ is shown by a black dotted line, identified by $|t|^{\lambda_{1}}$. The slopes of these lines for panels (a), (b) and (c) are respectively 0.994, 0.994 and 1.000. These are then the $t<0$ transition exponents $\left(\lambda_{1}^{X,-}\right)$ for chemical potential, condensate fraction and specific heat respectively in the one dimensional harmonic oscillator trap. These values and the corresponding TE for $t>0$ are presented in Table II for the largest $N$ chosen.

We next repeat the calculations for a two-dimensional isotropic harmonic oscillator trap for $N=$ $1000,10000,100000$ and 1000000 . In order to save space, we refrain from presenting plots for the 2-D case. In this case, $C_{N}(T)$ versus $T$ curve has a maximum, from which we calculate $T_{c}$ using Eq. (12). The asymptotic part of the plots similar to Fig. 3 are again found to be straight lines, which are parallel for different $N$ of the same panel, indicating that the transition exponents are independent of $N$. Calculated TE for both $t<0$ and $t>0$ are presented in the second row of Table II. 


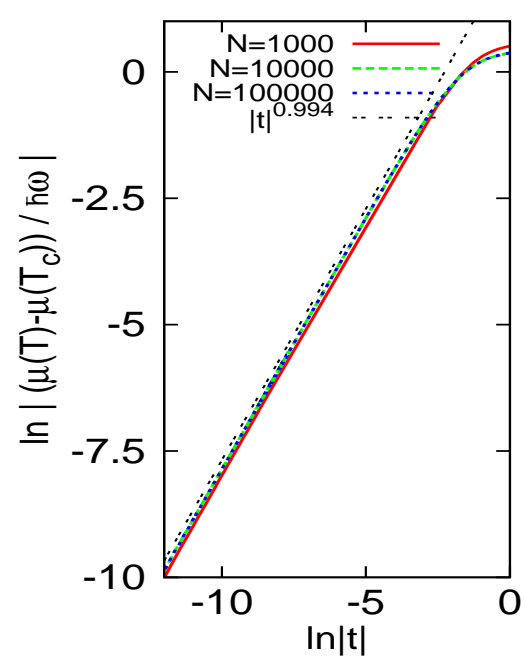

(a)

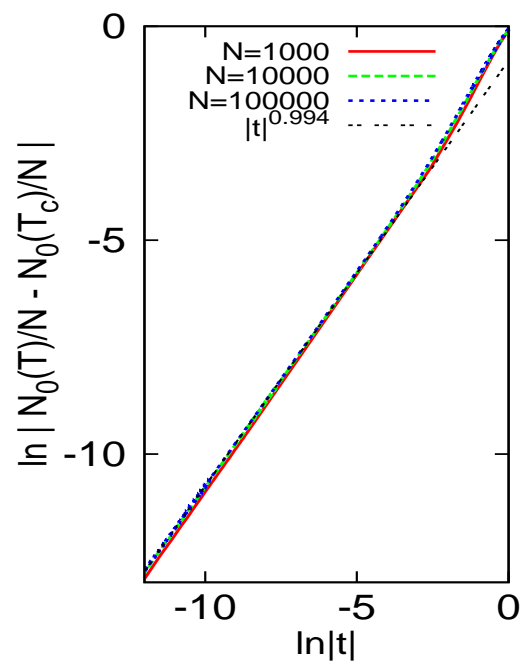

(b)

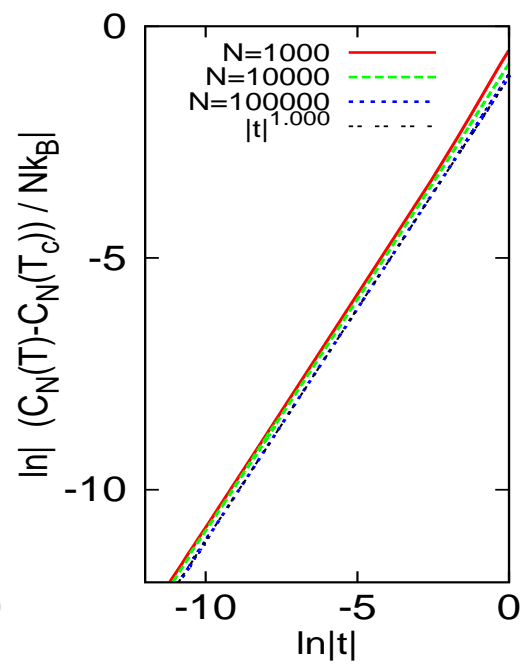

(c)

Figure 3: (Color online) Plot of $\ln \left|\left(\mu(T)-\mu\left(T_{c}\right)\right) / \hbar \omega\right|$ (panel a), $\ln \left|N_{0}(T) / N-N_{0}\left(T_{c}\right) / N\right|$ (panel b) and $\ln \left|\left(C_{N}(T)-C_{N}\left(T_{c}\right)\right) / N k_{B}\right|$ (panel c) as functions of $\ln |t|$ for indicated number $(N)$ of non-interacting bosons in a one-dimensional harmonic trap. Typical straight line fits to the asymptotic regions are shown by black dotted lines, indicated as $|t|^{\lambda_{1}}$, giving the best fit value of $\lambda_{1}$. Note that the asymptotic linear regions for different $N$ are parallel, showing that the transition exponent of a particular thermodynamic function is independent of $N$.

We next calculate these quantities for non-interacting bosons in a three dimensional trap. In this case also, $C_{N}(T)$ shows a maximum, hence we calculate $T_{c}$ using Eq. (12). In Fig. 4, we plot $\mu / \hbar \omega$, condensate fraction and $C_{N} / N k_{B}$ as functions of $T / T_{c}$ in panels (a), (b) and (c) respectively. Calculated values of $T_{c} / T_{c}^{0}$ are $0.896,0.949,0.976$, and 0.989 for $N=1000,10000,100000$ and 1000000 respectively, where $T_{c}^{0}$ is the critical temperature in the semi-classical treatment. The small difference between $T_{c}$ and $T_{c}^{0}$ is attributed to the fact that in the exact numerical calculation, $\mu$ is allowed to take appropriate values for $T<T_{c}$, instead of fixing it to be zero for $T \leq T_{c}^{0}$.

From plots (not presented) similar to those in Fig. 3, we calculate TE for both $t<0$ and $t>0$ for the three thermodynamic functions $(X)$ for all chosen values of $N$. Once again, we find the linear parts for all $N$ are parallel in the case of a given $X$. This again demonstrates that TE for a particular $X$ is independent of $N$. Calculated transition exponents for the largest $N$ have been listed in the third row of Table II. 


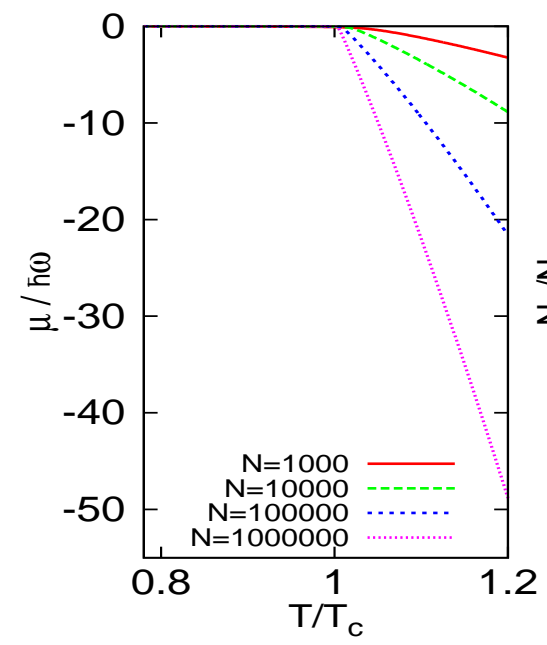

(a)

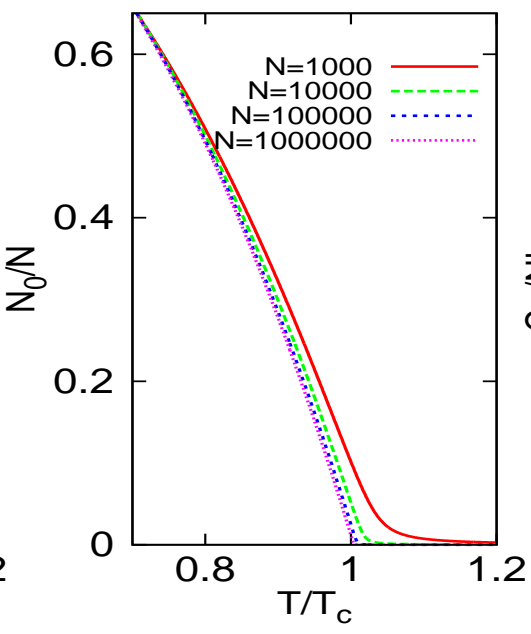

(b)

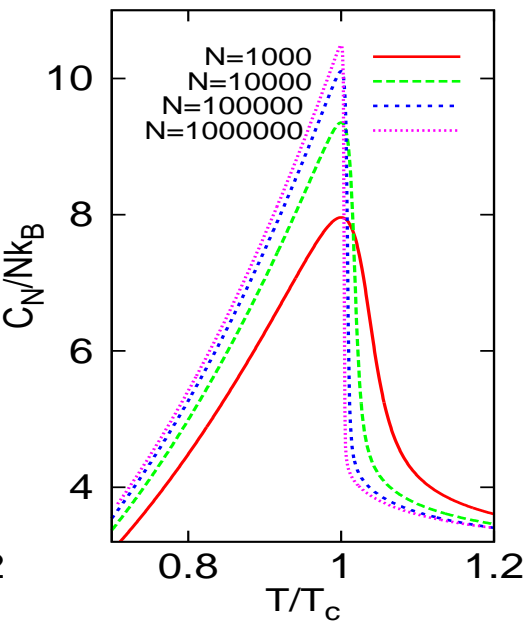

(c)

Figure 4: (Color online) Plot of $\mu / \hbar \omega$ (panel a), condensate fraction (panel b) and $C_{N} / N k_{B}$ (panel c) as functions of $T / T_{c}$ for indicated number $(N)$ of non-interacting bosons in a three-dimensional isotropic harmonic trap. In contrast with Fig. 2(c), panel (c) above shows a maximum followed by a sharp drop in $C_{N}$, the sharpness of the drop increasing with $N$. Furthermore, as $N \rightarrow \infty$, the temperature at which this sharp drop occurs approaches $T_{c}^{0}$.

In general, it is seen that as $\ln |t|$ increases, the plots belonging to a particular $X$ for different $N$ separate gradually, showing that the nature of the thermodynamic quantity away from the QPT region depends on $N$. On the other hand, numerical errors start to show up for large negative $\ln |t|$. Hence such points are ignored for calculation of TE. Numerical errors are larger for the calculation of the specific heat, since it involves many sums, differences and divisions [see Eq. (11)]. Corresponding plots show some divergences.

\section{III.2 Interacting bosons}

Next we present the results of our calculation for $N$ bosons interacting via the van der Waals potential and trapped in an isotropic three dimensional harmonic oscillator potential. As mentioned earlier, the GPE uses a contact interaction, whose strength depends only on the $s$-wave scattering length $a_{s}$ and hence is shape independent of the two-body potential. An earlier calculation [23] showed that calculated observables are indeed shape dependent. Hence it is appropriate that a finite-range realistic 
Table 2: Transition exponents for chemical potential, condensate fraction and specific heat of BEC for non-interacting bosons in one, two and three dimensions, as also bosons interacting through van der Waals potential and trapped by three-dimensional harmonic oscillator potential.

\begin{tabular}{|c|c|c|c|c|c|c|c|c|}
\hline \multirow{2}{*}{$\begin{array}{c}\text { Type of } \\
\text { BEC }\end{array}$} & \multirow[t]{2}{*}{$N$} & \multirow[t]{2}{*}{$\frac{k_{B} T_{c}}{\hbar \omega}$} & \multicolumn{3}{|c|}{$\lambda_{1}$ for $t<0$} & \multicolumn{3}{|c|}{$\lambda_{1}$ for $t>0$} \\
\hline & & & $\lambda_{1}^{\text {chempot, }-}$ & $\lambda_{1}^{\text {condfrac, }-}$ & $\lambda_{1}^{\text {spht, }-}$ & $\lambda_{1}^{\text {chempot, }+}$ & $\lambda_{1}^{\text {condfrac, }+}$ & $\lambda_{1}^{\mathrm{spht},+}$ \\
\hline $\begin{array}{c}\text { 1-D } \\
\text { non } \\
\text { interacting }\end{array}$ & $10^{5}$ & 10713.561 & 0.994 & 0.994 & 1.000 & 1.007 & 1.007 & 0.995 \\
\hline $\begin{array}{c}2-\mathrm{D} \\
\text { non } \\
\text { interacting }\end{array}$ & $10^{6}$ & 773.259 & 0.945 & 1.003 & 1.790 & 1.055 & 0.995 & 2.153 \\
\hline $\begin{array}{c}3-\mathrm{D} \\
\text { non } \\
\text { interacting }\end{array}$ & $10^{6}$ & 93.559 & 0.926 & 0.987 & 1.783 & 1.079 & 1.014 & 2.049 \\
\hline $\begin{array}{c}3-\mathrm{D} \\
\text { interacting } \\
\text { (vdW pot) }\end{array}$ & $5 \times 10^{3}$ & 7.650 & 1.032 & 1.004 & 1.878 & 1.005 & 0.991 & 2.041 \\
\hline
\end{tabular}

interatomic potential like the vdW potential should be used. Calculated values of $\ln \left|\left(\mu(T)-\mu\left(T_{c}\right)\right) / \hbar \omega\right|$, $\ln \left|\frac{N_{0}(T)}{N}-\frac{N_{0}\left(T_{c}\right)}{N}\right|$ and $\ln \left|C_{N}(T)-C_{N}\left(T_{c}\right)\right|$ [12] have been plotted against $\ln |t|$ in panels (a), (b) and (c) of Fig. 5. Once again these plots are straight lines in the asymptotic (ln $|t|$ large negative) region. The lower limit of $\ln |t|$ has been restricted to -7 to eliminate numerical errors, which enter inevitably for the interacting case. Plots of different $N$, belonging to a particular $X$ are again found to be parallel, showing that TE is independent of $N$. Calculated TE are presented in the fourth row of Table II.

Calculation of transition exponent involves logarithms of differences of quantities, for very small changes in $T$. Hence, allowing for relatively large errors in the calculation, we see from Table II that the transition exponents for chemical potential and condensate fraction, for both above and below the transition temperature are 1 for all cases studied. It is also 1 for the heat capacity in the one dimensional non-interacting case. For heat capacity in two and three dimensions, the transition exponent is 2 above the transition point and is about 1.8 below it. We already noticed that TE does not depend on the number of bosons in the trap. Furthermore, TEs for interacting bosons in 3D trap are found to be the same (within numerical errors) as the corresponding TE for the non-interacting bosons. These show 


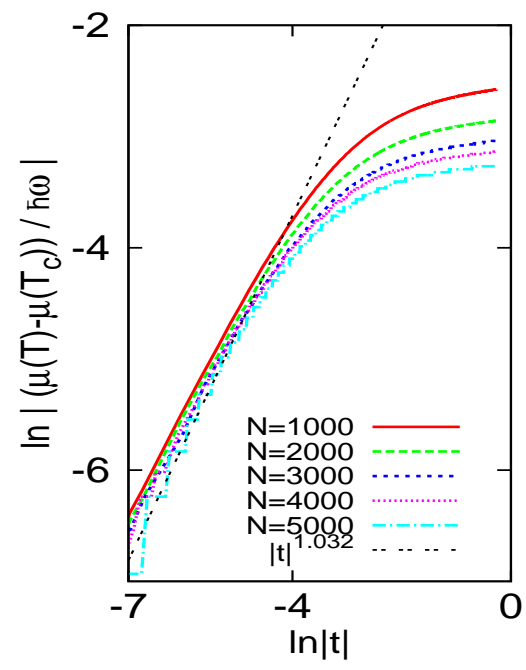

$\ln |\mathrm{t}|$

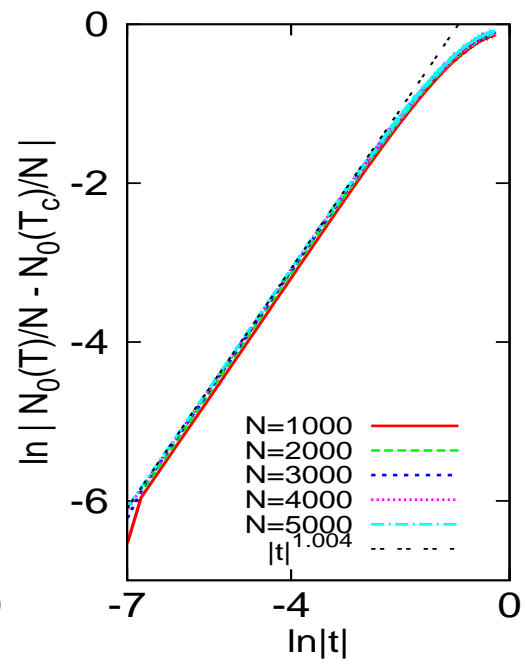

(b)

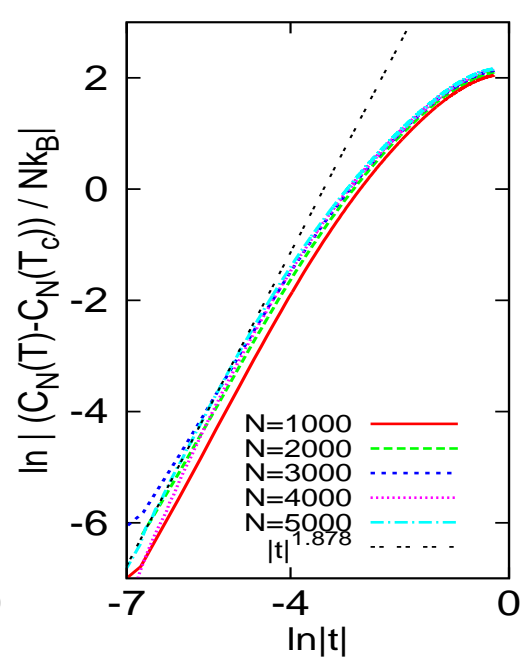

Figure 5: (Color online) Plot of $\ln \left|\left(\mu(T)-\mu\left(T_{c}\right)\right) / \hbar \omega\right|$ (panel a), $\ln \left|N_{0}(T) / N-N_{0}\left(T_{c}\right) / N\right|$ (panel b) and $\ln \left|\left(C_{N}(T)-C_{N}\left(T_{c}\right)\right) / N k_{B}\right|$ (panel c) as functions of $\ln |t|$ for indicated number of atoms interacting via van der Waals potential in a three-dimensional harmonic trap. For other comments, see caption of Fig. 3.

that the transition exponents may depend on the dimensionality of the system, but not on whether the bosons interact or not. Note that although the actual thermodynamic quantities near the transition region depend strongly on $N$ and whether the bosons are interacting or not, their intrinsic functional nature given by the transition exponent, as the transition temperature is approached, has universal characteristics.

From actual plot of thermodynamic quantities (see Figs. 2 and 4), one can notice that chemical potential and condensate fraction in all cases studied and $C_{N}(T)$ for one dimensional condensate are smooth functions even for very large $N$. A simple calculation shows that TE in such cases should be 1, as we found. On the other hand a value of 2 for the TE for $C_{N}(T)$ (for $T>T_{c}$ ) in two and three dimensions indicates a maximum. For $T<T_{c}$, TE has a fractional value between 1 and 2 . This means that the second derivative of $C_{N}(T)$ appears to diverge at $T \rightarrow T_{c}$ from below. However the result depends on the accuracy of numerical calculation. These behaviors are distinctly different from those obtained from the semi-classical treatment. 


\section{Conclusions}

In conclusion, we remark that a continuous quasi phase transition occurs in a non-interacting Bose gas trapped in a harmonic oscillator potential even in one-dimension. This is in sharp contrast with standard text book results [1, 4, but in agreement with Ketterle and van Druten [10. This is because the replacement of sums over single particle states by an integral over energy is not a valid approximation for discrete energy levels, especially when $N$ is small. Moreover, the assumption that $\mu=0$ for $T \leq T_{c}$ is not strictly valid in this case. However, we find that there are some distinct characteristic features in the one-dimensional case. For example, the specific heat is a monotonically increasing function, whereas in higher dimensions it has a maximum. However $C_{N}(T)$ is separately linear with different slopes for $T$ below and above $T_{c}$, exhibiting a transition behavior. But, as $N \rightarrow \infty$, the difference of these slopes tend to vanish. Thus the transition characteristics exhibited by the heat capacity gets wiped out in the large $N$ limit. On the other hand, chemical potential and condensate fraction continue to show QPT in this limit. The dependence of chemical potential, condensate fraction and specific heat on temperature for the non-interacting one-dimensionally trapped Bose gas has similar behavior as when a Bose gas interacts through the harmonic Calogero interaction [24]. The observation that the heat capacity becomes a smooth monotonically increasing function of $T$, with difference of slopes gradually decreasing in the large $N$ limit agrees with the commonly accepted idea that there is no Bose-Einstein condensation in one-dimension. Ketterle and van Druten argued that a quasi one-dimensional experimental setup is advantageous, since $T_{c}$ becomes larger [10. However, in view of the above discussion, it is clear that a quasi phase transition in $C_{N}(T)$ will be obscure in such an experimental setup with a large $N$.

We have also calculated the transition exponents for three thermodynamic functions, viz. chemical potential, condensate fraction and specific heat at constant particle number for a non-interacting Bose gas in an isotropic harmonic trap in one, two and three dimensions. For the three dimensional case, we have also investigated bosons interacting through a realistic two-body interaction. We find that the transition exponent for a particular thermodynamic function does not depend on $N$ or whether the bosons are interacting or not. It depends on the type of the thermodynamic function and the dimensionality of the space. This is consistent with the idea of universality. Even though the value of thermodynamic quantities depend strongly on $N$ and whether the bosons are interacting or not, besides the dimension of the space, their behavior near the transition point has a universal character. It is independent of $N$ and whether or not the bosons interact. 
We would like to thank Dr. Parongama Sen for drawing our attention to the critical exponent and universality, as well as for useful discussions. SG acknowledges CSIR (India) for a Senior Research Fellowship (Sanction No. : 09/028(0762)/2010-EMR-I). TKD acknowledges DST (India) for financial assistance through the USERS program.

\section{References}

[1] K. Huang, Statistical Mechanics (2nd Ed.), Wiley, New York (1987).

[2] F. Dalfovo, S. Giorgini, L. P. Pitaevskii and S. Stringari, Rev. Mod. Phys. 71463 (1999).

[3] R. K. Pathria, Statistical Mechanics (2nd Ed.), Butterworth-Heinemann, Oxford (1996).

[4] C. J. Pethick and H. Smith, Bose-Einstein Condensation in Dilute Gases, Cambridge University Press, Cambridge (2002).

[5] V. Bagnato, D. E. Pritchard and D. Kleppner, Phys. Rev. A 35, 4354 (1987).

[6] J. L. Roberts et al, Phys. Rev. Lett. 86, 4211 (2001).

[7] S. Stringari, Phys. Rev. Lett. 77, 2360 (1996).

[8] V. Bagnato and D. Kleppner, Phys. Rev. A 44, 7439 (1991).

[9] V. I. Yukalov, Phys. Rev. A 72, 033608 (2005).

[10] W. Ketterle and N. J. van Druten, Phys. Rev. A 54, 656 (1996).

[11] R. Napolitano, J. De Luca, V. S. Bagnato and G. C. Marques, Phys. Rev. A 553954 (1997).

[12] A. Biswas, J. Phys. B 42, 215302 (2009).

[13] J. M. Yeomans, Statistical Mechanics of Phase Transitions (Clarendon Press, Oxford, New York, 1992).

[14] T. K. Das, S. Canuto, A. Kundu and B. Chakrabarti, Phys. Rev. A 75, 042705 (2007).

[15] D. Blume and C. H. Greene, Phys. Rev. A 63, 063601 (2001).

[16] A. Kundu, B. Chakrabarti, T. K. Das and S. Canuto, J. Phys. B, 40, 2225 (2007).

[17] T. K. Das, B. Chakrabarti, Phys. Rev. A 70, 063601 (2004).

[18] B. Chakrabarti, A. Kundu and T. K. Das, J. Phys. B 38, 2457 (2005); A. Biswas and T. K. Das, J. Phys. B 41, 231001 (2008); B. Chakrabarti, T. K. Das and P. K. Debnath, Phys. Rev. A 79, 053629 (2009); T. K. Das, A. Kundu, S. Canuto and B. Chakrabarti, Phys. Lett. A, 373, 258 (2009). 
[19] J. L. Ballot and Fabre de la Ripelle, Ann. Phys. (N.Y.) 127, 62 (1980).

[20] M. Fabre de la Ripelle, Ann. Phys. (N.Y.) 147, 281 (1983).

[21] M. Abramowitz and I. A. Stegun, Handbook of Mathematical Functions, Dover Publications Inc., New York (1972).

[22] T. K. Das, H. T. Coelho and M. Fabre de la Ripelle, Phys. Rev. C 26, 2281 (1982).

[23] B. Chakrabarti and T. K. Das, Phys. Rev. A 78, 063608 (2008).

[24] B. Chakrabarti and T. K. Das, Phys. Rev. A 81, 015601 (2010). 